\title{
Acute respiratory infections in children from an ENT perspective
}

A. ZAMFIR-CHIRU-ANTON ${ }^{1}$, N. MANEA ${ }^{2,3}$, D.C. GHEORGHE ${ }^{2,3}$

${ }^{1}$ "Grigore Alexandrescu“ Clinical Hospital for Children, Bucharest

2 „M.S. Curie“ Emergency Clinical Hospital for Children, Bucharest

${ }^{3}$ "Carol Davila" University of Medicine and Pharmacy, Bucharest

\begin{abstract}
Background. Adenoidectomy remains a frequent surgical approach for recurrent upper respiratory tract infections (URTI) in children. We research if only age and collectivity are responsible for recurrences, or other anatomic conditions can account for the history of the patient.

Design. Retrospective case series review.

Material and method. Questionnaire about respiratory symptoms and correlations observed between the history of the patient and the presence or absence of short soft palate.

Results. 102 children could provide enough data to be included in the study. Frequent URTI were observed in 30\% of the affected children and only 16\% of their normal peers. 41\% of URTIs each persisted longer than 10 days in short soft palate children vs. $17 \%$ in normal patients. Broncho-pulmonary complications were encountered in $44 \%$ of children with short palate as opposed to $27 \%$ in others.

Conclusions. Short velli palatini can disturb normal swallowing process and allow rhinopharyngeal chronic inflammation. That can lead to persistent recurrent URTIs, more probable chronic obstruction through the adenoids and other diseases in pediatric ENT practice.
\end{abstract}

Keywords: adenoidectomy, URTIs, short soft palate

Acute respiratory infections in children are extremely common. Some studies report up to 9-10 episodes/year in the range of 1-6 years old. (1) Some conditions can be made responsible for this epidemiologic behaviour: season, crowding, colectivities, age-associated immunity. The complications arising from their incidence vary: otitis, tracheobronchial disease, meningitis.

Implications of acute respiratory infections in children are multiple: parental stress, overloading of the medical system and abusive/nonjudicious use of antibiotics (by medical staff) with consequent antimicrobial resistance. (2)
The issues about acute respiratory infections regard their ethiology and the most efficient/ right way to address their management or prevention. Parental concern and consequent low productivity are other aspects of upper respiratory tract infections (URTI) in children.

From an epidemiological point of view, social conditions are of utmost concern, because aerial contagion and colectivities can not be completely avoided. (3) Poor social environment and low medical addressability are not dependant on medical care and their solution lies in the community. 
The goal of this paper is to possibly demonstrate/hypothesize other (e.g. anatomical) factors contributing to recurrent acute respiratory infections in children, with arguments from the experience of our pediatric ENT department.

\section{MATERIAL AND METHOD}

102 children admitted into our pediatric ENT department for adenoidectomy, between January 2011 - December 2012, were enrolled in the study. An informed consent was obtained from each parent.

Every parent of the patients enrolled was asked to fill a questionnaire, regarding the upper respiratory infection history of their children. The addressed issues were: number of episodes of URTI/month, length of URTI (less or more than 10 days), broncho-pulmonary complications associated with URTI and cough as a persistent symptom among other symptoms of the acute respiratory disease. We introduced the last mentioned issue only later in the study, so only 64 patients answered the question regarding cough.

The children were admited and operated in our department, with adenoidectomy performed, by Beckmann curette technique, under general anesthesia. Local appearance of the pharynx, uvula and velli palatini was noted visually by the senior surgeon and recorded into the medical data. The soft palate was appreciated as being short or normal, subjectively. Figure 1 shows a typical aspect, with large distance from the velli palatini to posterior pharyngeal wall and bifid uvula.

The recorded data were used to assess the incidence of short soft palate in adenoidectomy patients. The association of specific URTI symptoms with this anatomic condition were researched.

\section{RESULTS}

Data obtained from the questionnaires are presented in Table 1 and 2.

The incidence of frecquent URTI (more than 1 episode each month) in adenoidectomy children with short soft palate is $29.4 \%$ as compared to $16 \%(\chi 2$-test $=0,016)$ in normal peers. The difference is statistically significant.

In $41 \%$ of the adenoidectomy patients with short velli palatini, duration of each URTI was longer than 10 days, versus $17 \%$ in normal children ( $x 2$-test $=0,0003)$, also significantly different.

Broncho-pulmonary complications and cough dominate clinical symptoms and disease in $44 \%$ of short palate patients but in only $18 \%$ for the others $(\chi 2=0,0001)$. Persistent cough was seen predominantly in chidren having the palatal incompetence, even if not as overt malformation and other specific symptoms.

\section{DISCUSSIONS}

Nasal anatomical conditions can predispose to particular/prolonged courses of infectious diseases of the nose. The responsible mechanisms could be: chronic obstruction that leads to poorer clearance of nasal mucus, eventually promoting local bacterial growth. Consequent hypoxia of the nasal mucosa favors further ciliary damage. Local inflammatory disease of the nasal mucosa from other diseases can also contribute to recurent local bacterial infections.

It has been cited in the literature that one condition promoting recurrent infections of the upper airways is pharyngo-laryngeal reflux. (4) It does so by maintaining an inflamatory status of the rhinopharynx, as stated by some authors.

TABLE 1. SUmmarized data from patients' questionnaires

\begin{tabular}{|l|c|c|c|c|c|c|}
\hline & $\begin{array}{c}\text { No. of } \\
\text { children }\end{array}$ & $\%$ & $\begin{array}{c}\text { Short Soft } \\
\text { Palate }\end{array}$ & $\%$ & $\begin{array}{c}\text { Normal soft } \\
\text { palate }\end{array}$ & $\%$ \\
\hline Total & 102 & & 34 & & 68 & \\
\hline$>1$ URTI/month & 21 & $20 \%$ & 10 & $29.4 \%$ & 11 & $16 \%$ \\
\hline URTI with > 10 days duration each & 26 & $25 \%$ & 14 & $41 \%$ & 12 & $17 \%$ \\
\hline Broncho-pulmonary complications & 27 & $26 \%$ & 15 & $44 \%$ & 12 & $18 \%$ \\
\hline
\end{tabular}

TABLE 2. Data regarding cough as a persistent symptom in adenoidectomy patients

\begin{tabular}{|l|c|c|c|c|c|c|}
\hline & $\begin{array}{c}\text { No. of } \\
\text { children }\end{array}$ & $\%$ & $\begin{array}{c}\text { Short Soft } \\
\text { Palate }\end{array}$ & $\%$ & $\begin{array}{c}\text { Normal soft } \\
\text { palate }\end{array}$ & $\%$ \\
\hline Total & 64 & & 20 & & 44 & \\
\hline $\begin{array}{l}\text { Cough dominating the clinical } \\
\text { picture }\end{array}$ & 22 & $34 \%$ & 10 & $50 \%$ & 12 & $27 \%$ \\
\hline
\end{tabular}


Lack of normal continence of the natural muscular ring of the rhinopharynx, as seen in our short soft palate patients, could be responsible, at least in part, for such condition.

Being a tertiary referal and a large surgical pediatric ENT center, most of our surgery comprises adenoidectomy as a standard approach for chronic inflammatory conditions and obstruction of the nose. It is a common approach in pediatric ENT. (5) We found that in many children submmited to this kind of surgery, short palate is a common finding, more than its prevalence in normal population (6) (33,3\% from our adenoidectomy patients). Due to the risks that this anatomical condition predisposes postoperatively (velopharyngeal incompetence in selected cases after adenoidectomy) (7), we tried to evaluate the incidence of such children ongoing adenoidectomy and compared their ratio to the risk of the same malformation in the general population. The figures are statistically significant.

As long as the most common issues that are presented as main reasons to perform adenoidectomy, from a patient's history point of view, are frequent URTIs and the difficulty to manage them, the question about the role of short palate in this condition can be discussed.

In our study, the number of upper respiratory infections that a child could have before performing adenoidectomy in our department was higher in children presenting with a short palate ( $30 \%$ vs $16 \%$ ). Also, acute respiratory episodes with a longer duration (more than 10 days) had a higher incidence in these patients: $41 \%$ vs $17 \%$ than in their normal pharyngeal counterparts. That figures suggest that an abnormal status of the nasal/ nasopharyngeal mucosa favors long and reccurent relapses of acute respiratory infectious disease.

The incidence of pulmonary complications associated with URTI was significantly higher in short palate children ( $44 \%$ vs $18 \%$ ). Cough, as a main symptom, was also seen in $50 \%$ abnormal children vs $27 \%$ in normal ones.

Adenoid obstruction of the posterior nasal passage can promote less clearing of the nasal secretions and enhance microbial inflammation and proliferation. Factors that can add more inflammatory reaction to the naso-pharyngeal mucosa have additional favorable effect on nasal persistent infections.

Cough can also be maintained by persistent discharge in the rhinopharynx. Every pathologic condition maintaining this situation can contribute to cough prolongation beyond normal healing of a simple infectious process.

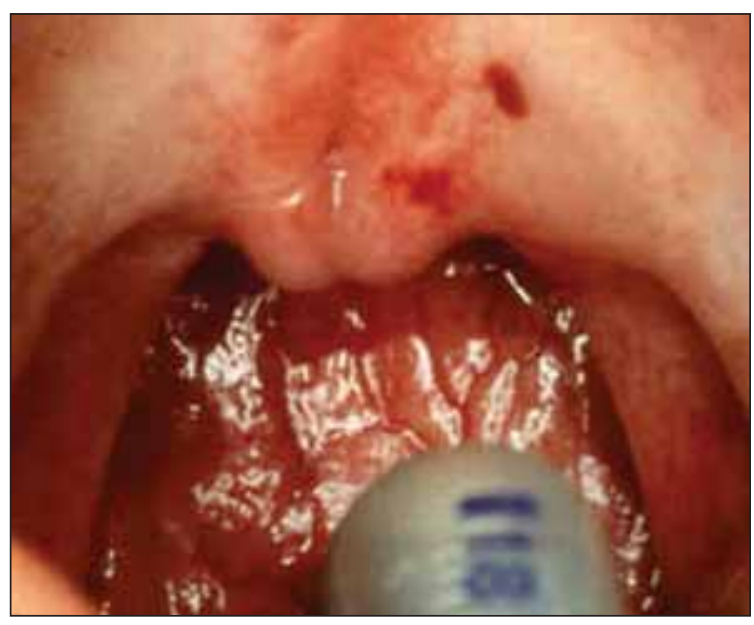

FIGURE 1. Large distance between uvula and posterior pharyngeal wall; bifid uvula

We hypothesize that the presence of a short velli palatini prediposes to partial reflux of food into the rhinopharynx, during deglutition. Mechanical and and chemical inflammation of rhinopharyngeal mucosa stimulates local pathogenic microbial flora. That is why these children are frequently seeking medical ent care or are submitted to adenoidectomy more frequently than others.

Some sorces of error can be questioned in our study. First of all, there is no definition of short palate in terms of anatomic measurements. (8) It can be demonstrated usually by means of nasal endoscopy on a cooperating child, showing a lack of complete closure of the rhinopharynx in certain types of phonation. Still, in a patient with a large obstructive adenoid pad, that vellar incompetence can be difficult to prove/show. (9) It is also impossible to predict postoperatively the distance between the soft palate and the oropharyngeal wall, after adenoidectomy. Instead, an experienced surgeon can be aware of the condition and appreciate "on the move" the existence of the condition. Usually, sophisticated images are not currently used for evaluation of an indication for adenoidectomy. Bifid uvula can be of use when noted clinically, but it is not always possible to detect it, especially in small or uncooperative children. Palpation of the palatal plate can bring sometimes information about an occult submucosal palatine cleft. (10)

\section{CONCLUSION}

Although there is no solution to this anatomical inherited condition, the specialist that observes a short soft palate can predict and warn 
the patient of possible frequent URTIs in spite of adenoidectomy. He can also propose partial adenoidectomy (e.g. shaver technique) in those cases where nasal obstruction is the only/main complaint and the risk of velopharyngeal insufficiency is high.

\section{REFERENCES}

1. Van den Aardweg M.T., et al. Effectiveness of adenoidectomy in children with recurrent upper respiratory tract infections: open randomised controlled trial. BMJ, 2011. 343: p. d5154.

2. Carabin H., et al. Estimation of direct and indirect costs because of common infections in toddlers attending day care centers. Pediatrics, 1999. 103(3): p. 556-64.

3. Harsten G., et al. Acute respiratory tract infections in children. A three-year follow-up from birth. Acta Paediatr Scand, 1990. 79(4): p. 402-9.

4. Monroy A., Behar P., Brodsky L. Revision adenoidectomy--a retrospective study.
Int J Pediatr Otorhinolaryngol, 2008. 72(5): p. 565-70.

5. Kvaerner K.J., Nafstad P., Jaakkola J.J. Otolaryngological surgery and upper respiratory tract infections in children: an epidemiological study. Ann Otol Rhinol Laryngol, 2002. 111(11): p. 1034-9.

6. Gibb A.G. Hypernasality (rhinolalia aperta) following tonsil and adenoid removal. J Laryngol Otol, 1958. 72(6): p. 433-51.

7. Saunders N.C., et al. Velopharyngeal insufficiency following adenoidectomy. Clinical otolaryngology and allied sciences, 2004. 29(6): p. 686-8.
8. Rudnick E.F., Sie K.C. Velopharyngeal insufficiency: current concepts in diagnosis and management. Current opinion in otolaryngology \& head and neck surgery, 2008. 16(6): p. 530-5.

9. Ibuki K., Karnell M.P., Morris H.L. Reliability of the nasopharyngeal fiberscope (NPF) for assessing velopharyngeal function. Cleft Palate J, 1983. 20(2): p. 97-107.

10. Kaplan E.N. The occult submucous cleft palate. The Cleft palate journal, 1975. 12: p. $356-68$. 